\title{
On radical classes of monounary algebras
}

\author{
Zuzana Farkasová and Danica \\ Jakubiková-Studenovská
}




\title{
ON RADICAL CLASSES OF MONOUNARY ALGEBRAS
}

\author{
ZUZANA FARKASOVÁ AND DANICA JAKUBÍKOVÁ-STUDENOVSKÁ \\ Received 10 October, 2012
}

\begin{abstract}
Our work treats monounary algebras and their radical classes with respect to their homogeneity. Homogeneous radical classes and homomorphism-homogeneous radical classes of monounary algebras are characterized. Further, torsion classes of monounary algebras are described.
\end{abstract}

2010 Mathematics Subject Classification: 08A60; 08C99

Keywords: monounary algebra, homogeneous algebra, homomorphism-homogeneous algebra, homogeneous radical class, homomorphism-homogeneous radical class

\section{INTRODUCTION}

A major theme in universal algebra is the study of classes of algebras of the same type that are closed under one or more constructions [2]. For algebraic structures of a given type it might be useful to investigate various classes (e.g., varieties, torsion classes, radical classes, convexities) in order to classify the structures. By the classification we mean the division of algebraic structures into classes, according to their common features.

The general study of varieties of algebras with finitary operations was developed by G. Birkhoff in the 1930's. B.H. Neumann initiated the investigation of varieties of groups [20]. In the 70's J. Martinez began the study of varieties of $\ell$-groups [16] and later he studied also torsion classes of $\ell$-groups [17]. Further, torsion theory for acts (semigroup action on sets) was developed in [15]. Radical classes, as a generalization of torsion classes, of lattice ordered groups were introduced by J. Jakubík [8]. These classes were explored for different types of operators. Some other results for radical classes of $\ell$-groups are found in [5,25], for radical classes of generalized Boolean algebras in [9] and for radical classes of MV-algebras are available in [10].

The notion of homogeneity has been applied in different fields of mathematics in connection with deep results. As examples, it suffices to mention the investigation of

Supported by VEGA Grant 2/0194/10 and VVGS-PF-2012-50, respectively. 
continuous geometries [26] or the study of homogeneous Boolean algebras [22]. Cameron and Nešetřil [3] introduced the notion of homomorphism-homogeneous structures; this notion is closely related to the classical notion of homogeneity. The importance of the notion of homomorphism-homogeneity for algebras is considerable, see e.g., [6,13], or for relational structures [18, 19,23].

Some properties of algebraic structures of a finite type can be described by means of analogous properties of certain assigned monounary algebras. So monounary algebras play a significant role in the study of algebraic structures (cf. monographs $[4,7,12])$. The advantage of monounary algebras is their relatively simple visualization, since they can be represented by oriented planar graphs. Monounary algebras are often considered as special types of automata [1,21]. Also, they were studied in connection with other algebraic structures. For example, monounary algebras enriched with certain relations have been studied in [14,24].

Several classes of monounary algebras (e.g., varieties, retract varieties, convexities) were described in [7]. The aim of this work is a description of radical classes of monounary algebras, where the radical classes are considered with respect to two types of homogeneity. Namely, we characterize homogeneous radical classes and homomorphism-homogeneous radical classes. In the end we deal also with homogeneous torsion classes and homomorphism-homogeneous torsion classes of monounary algebras.

\section{BASIC NOTATIONS AND DEFINITIONS}

Let $\mathbb{Z}$ be the set of all integers, $\mathbb{N}$ be the set of all positive integers and $\mathbb{N}_{0}=$ $\mathbb{N} \cup\{0\}$. Next, let us denote by $C$ ard the system of all cardinal numbers.

For the basic notions and definitions concerning monounary algebras we refer to [7]. Also, we will apply notions and definitions from [13]; let us recall some of them.

Definition 1. A monounary algebra is a pair $(A, f)$ where $A$ is a non-empty set and $f: A \rightarrow A$ is a unary operation on $A$.

Notice that we will consider the empty set $\varnothing$ as a monounary algebra and instead of $(A, f)$ we will often write $A$ as an abbreviation.

Let $(A, f)$ be a monounary algebra. Then for $x \in A$, put $f^{0}(x)=x$ and $f^{-1}(x)=$ $\{y \in A: f(y)=x\}$. If for some $n \in \mathbb{N}, f^{n-1}(x)$ is defined, then we set $f^{n}(x)=$ $f\left(f^{n-1}(x)\right)$.

An algebra $(A, f)$ is called connected, if for each $x, y \in A$ there exist $m, n \in \mathbb{N}_{0}$ such that $f^{m}(x)=f^{n}(y)$.

A set $B \subseteq A$ is called a connected component of $(A, f)$, if $B$ is a maximal connected subalgebra of $A$.

Let $x \in A$ be such that there exists $m \in \mathbb{N}$ with $f^{m}(x)=x$. Then $x$ is called a cyclic element of $A$. If such $m \in \mathbb{N}$ does not exist then we say that $x$ is acyclic. Let $n \in \mathbb{N}$ be the smallest integer such that $f^{n}(x)=x$. Then the set of elements 
$C=\left\{x, f(x), \ldots, f^{n-1}(x)\right\}$ is called a cycle of $(A, f)$ and $n$ is called the length of $C$; so $C$ is an $n$-element cycle of $A$.

We denote by $\mathbb{Z}=(\mathbb{Z}, f)$ a monounary algebra such that $f(x)=x+1$ for all $x \in \mathbb{Z}$. Next, for $n \in \mathbb{N}$, let $\mathbb{Z}_{n}=\left(\mathbb{Z}_{n}, f\right)$ be a monounary algebra such that $\mathbb{Z}_{n}=$ $\{0,1, \ldots n-1\}$ and $f(i)=i+1(\bmod n)$ for each $i \in \mathbb{Z}_{n}$.

For $\alpha \in \mathbb{C}$ ard $-\{0\}$ let $V_{\alpha}=\left(V_{\alpha}, f\right)$ be a connected monounary algebra possessing no cycle and such that $\left|f^{-1}(v)\right|=\alpha$ for every element $v \in V_{\alpha}$. We remark that such algebra exists for each $\alpha \in \mathbb{C}$ ard $-\{0\}$ and it is unique up to isomorphism.

Let $A_{i}, i \in I$ be a system of mutually disjoint algebras. Denote by $\sum_{i \in I} A_{i}$ a disjoint union of algebras $A_{i}, i \in I$. If there exists an algebra $A$ such that $A_{i} \cong A, i \in I$ and $|I|=k$, then instead of $\sum_{i \in I} A_{i}$ we write $k \cdot A$. Note that for $k=0(I=\varnothing), k \cdot A=\varnothing$ $\left(\sum_{i \in I} A_{i}=\varnothing\right)$.

Let $A=(A, f)$ be a monounary algebra. For a connected component $S \subseteq A$ let $\operatorname{cn}(S)$, the cycle number of $S$ denote the length of the cycle in $S$. If $S$ does not have any cycle, we $\operatorname{set} \operatorname{cn}(S)=\infty$.

We say that $a \in A$ is a source in $A$ if $f^{-1}(a)=\varnothing$.

A branch in a monounary algebra $A$ is a finite or infinite sequence $a_{1}, a_{2}, a_{3}, \ldots$ such that

- $a_{i}=f\left(a_{i+1}\right)$ for all $i \geq 1$,

- $a_{i}$ is acyclic for all $i \geq 1$, and

- if the sequence is finite, that if it has the form $a_{1}, a_{2}, a_{3}, \ldots, a_{n}$, then $a_{n}$ is a source in $A$.

For an acyclic element $a \in A$, the height of $a(\operatorname{ht}(a)$, for short) means the least number $k \geq 1$ such that $f^{k}(a)$ is a cyclic element. If no such $k$ exists, we set $\operatorname{ht}(a)=\infty$.

A connected monounary algebra $A$ is said to be regular if

- $A$ possesses a cycle, and

- either every branch in $A$ is infinite, or every branch in $A$ is finite and ht $(a)=$ ht $(b)$ for all sources $a, b$ in $A$.

Note that a connected algebra consisting of solely cyclic elements is regular.

If $A$ is a regular algebra, then the height of $A$, denoted by $\operatorname{ht}(A)$, is defined as follows:

- $\operatorname{ht}(A)=0$ if there are no acyclic elements in $A$ (i.e., $A$ is a cycle);

- $\operatorname{ht}(A)=\infty$ if every branch starting at a cyclic element of $A$ is infinite;

- otherwise, $\operatorname{ht}(A)$ denotes the common height of all the sources in $A$.

Definition 2. A monounary algebra $(A, f)$ is said to be homogeneous if for each $x, y \in A$ there is an automorphism $\varphi$ of $A$ such that $\varphi(x)=y$. 
Definition 3. We say that a monounary algebra $A=(A, f)$ is homomorphismhomogeneous if every homomorphism $\varphi: B \rightarrow A$ from a finitely generated subalgebra $B$ of $A$ into $A$ extends to an endomorphism of $A$.

We will consider the empty set $\varnothing$ as a homogeneous and homomorphism-homogeneous monounary algebra, too.

\section{Homogeneous RADiCAL ClASSES}

In this section we characterize classes of monounary algebras which are radical with respect to homogeneity. Homogeneous monounary algebras have been described in the paper [11].

Definition 4. A non-empty class $\mathcal{K}$ of monounary algebras is called a homogeneous radical class (hr-class, for short) if it satisfies the following conditions:

(0) $\mathcal{K}$ is closed with respect to isomorphisms,

(1) whenever $A \in \mathcal{K}$ and $B$ is a homogeneous subalgebra of $A$, then $B \in \mathcal{K}$,

(2) for each monounary algebra $D$ there exists its greatest homogeneous subalgebra belonging to $\mathcal{K}$.

Note that the condition (2) means that for each monounary algebra $D$, the system of all homogeneous subalgebras of $D$ belonging to $\mathcal{K}$ has a greatest element. The following theorem is proved in [11].

Theorem 1 ([11]). Let A be a monounary algebra. Then A is homogeneous if and only if either

(1) $A \cong \beta \cdot \mathbb{Z}_{n}$ for $\beta \in \mathbb{C}$ ard, $n \in \mathbb{N}$,

or

(2) $A \cong \beta \cdot \mathbb{V}_{\alpha}$ for $\alpha, \beta \in \mathbb{C}$ ard, $\alpha>0$.

Notice that the monounary algebra $\beta \cdot \mathbb{Z}_{n}$ is isomorphic to a monounary algebra consisting of $\beta$ cycles of length $n$.

We show some necessary conditions for a class $\mathcal{K}$ to be homogeneous radical. First, it is proved that an hr-class does not contain a monounary algebra possessing a subalgebra isomorphic to $\mathbb{Z}$.

Lemma 1. Let $\mathcal{K}$ be an hr-class of monounary algebras and $A$ be a monounary algebra such that some its subalgebra is isomorphic to $\mathbb{Z}$. Then the algebra A does not belong to the class $\mathcal{K}$.

Proof. By way of contradiction, suppose that $A \in \mathcal{K}$. Denote by $B$ a subalgebra of $A$ such that $B \cong \mathbb{Z}$. According to Theorem 1, $B$ is a homogeneous subalgebra of $A$. Thus by (1) of Definition $4, B \in \mathcal{K}$.

Let us consider a connected monounary algebra $D$ without cyclic elements, having the property that $\left|f^{-1}(x)\right|=2$ for only one element $x \in D$ and $\left|f^{-1}(y)\right|=1$ for 
each $y \in D-\{x\}$. Observe that $D$ has two maximal homogeneous subalgebras $D_{i}$ ( $i=1,2)$ belonging to the class $\mathcal{K}$ and $D_{i} \cong B(i=1,2)$. But $D_{i}(i=1,2)$ is not the largest homogeneous subalgebra of $D$ such that $D_{i} \in \mathcal{K}$. This contradicts the condition (2) of Definition 4 and completes the proof.

Lemma 2. Let $\mathcal{K}$ be a class of monounary algebras satisfying (0) and (1) of Definition 4. Further, let there exist $A, B \in \mathcal{K}$ and $m, n \in \mathbb{N}, m \neq n$ such that the algebra $A$ contains a subalgebra isomorphic to $\mathbb{Z}_{m}$ and the algebra $B$ contains $a$ subalgebra isomorphic to $\mathbb{Z}_{n}$. Then the class $\mathcal{K}$ does not satisfy (2) of Definition 4.

Proof. By the assumption, $\mathbb{Z}_{m}$ and $\mathbb{Z}_{n}$ belong to the class $\mathcal{K}$. Let us take a monounary algebra $D$ such that $D=\mathbb{Z}_{m}+\mathbb{Z}_{n}$. The algebras $\mathbb{Z}_{m}$ and $\mathbb{Z}_{n}$ are homogeneous subalgebras of $D$ and $\mathbb{Z}_{m}, \mathbb{Z}_{n} \in \mathcal{K}$. These subalgebras of $D$ are maximal but none of them is the largest homogeneous subalgebra of $D$ belonging to $\mathcal{K}$. Thus (2) of Definition 4 fails to hold.

Corollary 1. If a class $\mathcal{K}$ of monounary algebras satisfies (0) and (1) of Definition 4 and contains an algebra $A$ such that A possesses cycles of different lengths, then the class $\mathcal{K}$ does not satisfy (2) of Definition 4.

Proof. The assertion follows from Lemma 2 if we put $B=A$.

Lemma 3. Let $\mathcal{K}$ be an hr-class of monounary algebras. If $\mathbb{Z}_{n} \in \mathcal{K}$, then for each cardinal $\alpha, \alpha \cdot \mathbb{Z}_{n} \in \mathcal{K}$, too.

Proof. Assume that $\mathbb{Z}_{n} \in \mathcal{K}$ and suppose, to the contrary, that $\alpha \cdot \mathbb{Z}_{n} \notin \mathcal{K}$ for some $\alpha \in \mathbb{C}$ ard. Obviously, $\alpha \notin\{0,1\}$. By (2) of Definition $4, \alpha \cdot \mathbb{Z}_{n}$ contains a largest homogeneous subalgebra $X$ such that $X \in \mathcal{K}$. Since $\alpha \cdot \mathbb{Z}_{n} \notin \mathcal{K}, X$ is a proper subalgebra of $\alpha \cdot \mathbb{Z}_{n}$. Then there exists a homogeneous subalgebra $Y$ of $\alpha \cdot \mathbb{Z}_{n}$ such that $Y \cong \mathbb{Z}_{n} \in \mathcal{K}$ and $Y \nsubseteq X$. The subalgebra $Y$ of $\alpha \cdot \mathbb{Z}_{n}$ is incomparable with $X$ because $Y \nsubseteq X$. Hence $X$ cannot be the largest homogeneous subalgebra of $\alpha \cdot \mathbb{Z}_{n}$ such that $X \in \mathcal{K}$, which is a contradiction.

Theorem 2. Let $\mathcal{K}$ be a class of monounary algebras closed with respect to isomorphisms and let $\varnothing \in \mathcal{K}$. Then $\mathcal{K}$ is an hr-class if and only if either

(a) if $A \in \mathcal{K}$ then $A$ possesses neither cycle nor subalgebra isomorphic to $\mathbb{Z}$, or

(b) there exists $n \in \mathbb{N}$ such that

(i) $\alpha \cdot \mathbb{Z}_{n} \in \mathcal{K}$ for each $\alpha \in \mathbb{C}$ ard, and

(ii) if $A \in \mathcal{K}$ then $A$ possesses neither $m$-element cycle for $m \neq n$ nor subalgebra isomorphic to $\mathbb{Z}$.

Proof. Suppose that $\mathcal{K}$ is an hr-class. From Lemma 1 it follows that $\mathcal{K}$ does not contain an algebra possessing a subalgebra isomorphic to $\mathbb{Z}$. Next, we distinguish two cases:

Case 1: Let for each $A \in \mathcal{K}, A$ possess no cycle. According to Lemma 1, $A$ does not contain a subalgebra which is isomorphic to $\mathbb{Z}$. Hence, the condition (a) is satisfied. 
Case 2: Suppose that there exists an algebra $A_{0} \in \mathcal{K}$ such that $A_{0}$ possesses a subalgebra isomorphic to $\mathbb{Z}_{n}$ for some $n \in \mathbb{N}$. By Corollary $1, A_{0}$ does not contain any $m$-element cycle for $m \neq n$. Now take $A \in \mathcal{K}$. According to Lemma 2, $A$ possesses no subalgebra isomorphic to $\mathbb{Z}_{m}$ for $m \neq n$. Thus, condition (ii) of case (b) is valid. Observe that $\mathbb{Z}_{n} \in \mathcal{K}$ because $A_{0} \in \mathcal{K}$ and $\mathcal{K}$ satisfies (1) of Definition 4. So, condition (i) of case (b) follows from Lemma 3.

Next, we prove the converse implication of the theorem. The implication is trivial in the case (a). Now, assume that case (b) holds. By assumption, (0) of Definition 4 holds. Let $A \in \mathcal{K}$ and $B$ be a homogeneous subalgebra of $A$. According to Theorem $1, B \cong \beta \cdot \mathbb{Z}_{n}$ for some $\beta \in \mathbb{C}$ ard. Then by condition (i) of case (b), $B \in \mathcal{K}$. Hence, $\mathcal{K}$ satisfies (1) of Definition 4 . Let us consider an arbitrary monounary algebra $D$. If $D$ has no subalgebra isomorphic to $\mathbb{Z}_{n}$ then $\varnothing$ is the largest homogeneous subalgebra of $D$ such that $\varnothing \in \mathcal{K}$. Otherwise, denote by $E$ the sum of all subalgebras of $D$ isomorphic to $\mathbb{Z}_{n}$. Then $E$ is the largest homogeneous subalgebra of $D$, $E \cong \alpha \cdot \mathbb{Z}_{n}$ for some nonzero cardinal $\alpha$, thus $E \in \mathcal{K}$. This implies that $\mathcal{K}$ satisfies (2) of Definition 4. Therefore, $\mathcal{K}$ is an hr-class which concludes the proof.

For an hr-class $\mathcal{K}$ denote by $\mathscr{H}(\mathcal{K})$ the subclass of $\mathcal{K}$ such that $\mathscr{H}(\mathcal{K})$ consists of all homogeneous algebras of $\mathcal{K}$. Then $\mathscr{H}(\mathcal{K})$ is called a homogeneous part of $\mathcal{K}$. By Theorem $2, \mathscr{H}(\mathcal{K})$ is an hr-class.

\section{HOMOMORPHISM-HOMOGENEOUS RADICAL CLASSES}

The present section is devoted to the characterization of classes of monounary algebras which are radical with respect to homomorphism-homogeneity of monounary algebras.

The following theorem is proved in the paper [13].

Theorem 3 ([13]). Let A be a non-empty monounary algebra. Then $A$ is homomorphism-homogeneous if and only if A satisfies one of the following conditions:

(1) every branch in A is infinite,

(2) every connected component in $A$ is regular, and for any two connected components $S_{1}, S_{2} \subseteq A$, if $\operatorname{cn}\left(S_{1}\right) \mid \operatorname{cn}\left(S_{2}\right)$ then $\operatorname{ht}\left(S_{1}\right) \geq \operatorname{ht}\left(S_{2}\right)$ or ht $\left(S_{1}\right)=0$.

Definition 5. A non-empty class $\mathcal{K}$ of monounary algebras will be called a homomorphism-homogeneous radical class (for short, Hhr-class) if it satisfies the following conditions:

(0) $\mathcal{K}$ is closed with respect to isomorphisms,

(1) whenever $A \in \mathcal{K}$ and $B$ is a homomorphism-homogeneous subalgebra of $A$, then $B \in \mathcal{K}$,

(2) for each monounary algebra $D$ there exists its largest homomorphism-homogeneous subalgebra belonging to $\mathcal{K}$. 
From the above definition immediately follows

Corollary 2. Let $\mathcal{K}$ be a class of monounary algebras, closed with respect to isomorphisms and let $\varnothing \in \mathcal{K}$. Further, assume that each $A \in \mathcal{K}$ possesses neither cycle nor subalgebra isomorphic to $\mathbb{Z}$. Then $\mathcal{K}$ is an Hhr-class.

We start by showing some necessary conditions for a class $\mathcal{K}$ to be homomorphism-homogeneous radical.

Lemma 4. Let $\mathcal{K}$ be a class of monounary algebras satisfying (0) and (1) of Definition 5. Next, let $A$ be a regular monounary algebra such that $A$ has a unique branch and $\operatorname{ht}(A)=2$. If $A \in \mathcal{K}$ then $\mathcal{K}$ does not satisfy (2) of Definition 5.

Proof. Assume that $A \in \mathcal{K}$ and suppose, on the contrary, that $\mathcal{K}$ satisfies (2) of Definition 5. Denote by $B$ a regular subalgebra of $A$ such that ht $(B)=1$; and let $C$ be the cycle of $A$. Note that $B$ and $C$ are homomorphism-homogeneous subalgebras of $A$. Thus by (1) of Definition $5, B, C \in \mathcal{K}$. Let us consider a monounary algebra $D=D_{1}+D_{2}$ such that $D_{1} \cong A$ and $D_{2} \cong B$. We show that $D$ possesses no largest homomorphism-homogeneous subalgebra belonging to $\mathcal{K}$. This will lead to a contradiction. At first we make the following observations:

For $E=E_{1}+E_{2}$ where $E_{1} \cong E_{2} \cong B$, we get that $E_{1}, E_{2} \in \mathcal{K}$ according to (0) of Definition 5. Suppose that $E \notin \mathcal{K}$. Then $E$ possesses a largest homomorphismhomogeneous subalgebra belonging to $\mathcal{K}$, denote it by $E_{\mathcal{K}}$. Since $E_{\mathcal{K}}$ is a proper subalgebra of $E$ there exists an element $x \in E-E_{\mathcal{K}}$. Observe that there is a unique $i \in\{1,2\}$ with $x \in E_{i} \neq E_{\mathcal{K}}$. On the other hand, $E_{\mathcal{K}}$ is incomparable with $E_{i}$. Thus $E_{\mathcal{K}}$ cannot be the largest homomorphism-homogeneous subalgebra of $E$ belonging to $\mathcal{K}$, which is a contradiction. Hence, $E \in \mathcal{K}$.

Let $F=F_{1}+F_{2}$ where $F_{1} \cong A$ and $F_{2} \cong C$. Then $F_{1}, F_{2} \in \mathcal{K}$ according to (0) of Definition 5. Analogously as above we can show that $F \in \mathcal{K}$.

Note that $D$ is not a homomorphism-homogeneous algebra and that $D$ possesses two homomorphism-homogeneous subalgebras $X, Y$ such that $X \cong E$ and $Y \cong F$. So, $D$ contains two maximal homomorphism-homogeneous subalgebras belonging to $\mathcal{K}$, namely $X$ and $Y$, but none of them is the largest. This contradicts (2) of Definition 5 and completes the proof.

Corollary 3. Let $\mathcal{K}$ be a class of monounary algebras satisfying (0) and (1) of Definition 5 and $A$ be a monounary algebra possessing a regular subalgebra of height greater than 1. If $A \in \mathcal{K}$ then $\mathcal{K}$ fails to be an Hhr-class.

Proof. Assume that $A \in \mathcal{K}$ and suppose, to the contrary, that $\mathcal{K}$ is an Hhr-class. There exists a regular subalgebra $B$ of $A$ such that $B$ has a unique branch and $\operatorname{ht}(B)=$ 2. According to Theorem $3, B$ is a homomorphism-homogeneous algebra. Then from the assumption, $B \in \mathcal{K}$. In view of Lemma 4 , a class $\mathcal{K}$ does not satisfy (2) of Definition 5, a contradiction. 
Lemma 5. Let $\mathcal{K}$ be a class of monounary algebras satisfying (0) and (1) of Definition 5 and let $A$ be a regular monounary algebra of nonzero height. If $\mathbb{Z}, A \in \mathcal{K}$ then $\mathcal{K}$ does not satisfy (2) of Definition 5.

Proof. By way of contradiction, suppose that $\mathbb{Z}, A \in \mathcal{K}$ and $\mathcal{K}$ satisfies (2) of Definition 5. Corollary 3 implies that $\operatorname{ht}(A)<2$, hence $\operatorname{ht}(A)=1$. Consider an algebra $D=\mathbb{Z}+A$ which is not a homomorphism-homogeneous algebra in view of Theorem 3. According to (2) of Definition 5, $D$ possesses a largest homomorphismhomogeneous subalgebra belonging to $\mathcal{K}$, denote it by $D_{\mathcal{K}}$. Observe that $D_{\mathcal{K}}$ is a proper subalgebra of $D$, therefore there exists an element $x \in D-D \mathcal{K}$ and either $x \in$ $\mathbb{Z}$ or $x \in A$. By Theorem $3, \mathbb{Z}$ and $A$ are homomorphism-homogeneous subalgebras of $D$. Assume that $x \in \mathbb{Z}$ (or $x \in A$ ). Since $\mathbb{Z} \nsubseteq D \mathcal{K}$ (or $A \nsubseteq D \mathcal{K}$ ), $D_{\mathcal{K}}$ cannot be the largest homomorphism-homogeneous subalgebra of $D$ such that $D \mathcal{K} \in \mathcal{K}$. Hence, $D$ does not have a largest homomorphism-homogeneous subalgebra belonging to $\mathcal{K}$. This is a contradiction and completes the proof.

The following assertion follows from Lemmas 4 and 5.

Corollary 4. Let $\mathcal{K}$ be an Hhr-class containing an algebra possessing a regular monounary subalgebra of nonzero height. Then $\mathcal{K}$ does not contain an algebra with a subalgebra isomorphic to $\mathbb{Z}$ and does not contain an algebra possessing a regular subalgebra of height greater than one.

Next, if $\mathcal{K}$ is an Hhr-class such that $\mathbb{Z} \in \mathcal{K}$ then $\mathcal{K}$ fails to contain an algebra which possesses a regular subalgebra of nonzero height.

Notation 1 . Let for $n \in \mathbb{N}, Z_{n}^{1}$ be the class of all regular monounary algebras of height 1 and cycle number $n$. Next, let $Z_{n}=\left\{A: A \cong \mathbb{Z}_{n}\right\}$.

Clearly, if $\mathcal{K}$ is an Hhr-class such that $\mathcal{K} \cap \mathcal{Z}_{n} \neq \varnothing$ then $\mathcal{Z}_{n} \subseteq \mathcal{K}$. We show that whenever $\mathcal{K}$ is an Hhr-class such that $\mathcal{K} \cap \mathcal{Z}_{n}^{1} \neq \varnothing$ then $\mathcal{Z}_{n}^{1} \subseteq \mathcal{K}$.

Definition 6. Let $A \in Z_{n}^{1}$ for some $n \in \mathbb{N}$. Then the width of a cyclic element $a$ in $A$ denoted by w $(a)$ is defined as follows:

$$
\mathrm{w}(a)=\left|f^{-1}(a)\right|-1 .
$$

Lemma 6. Let $\mathcal{K}$ be an Hhr-class such that $\mathcal{Z}_{n}^{1} \cap \mathcal{K} \neq \varnothing$ for some $n \in \mathbb{N}$. Then for each $\beta \in \mathbb{C}$ ard $-\{0\}$ there exists an algebra $Y \in Z_{n}^{1} \cap \mathcal{K}$ such that $\mathrm{w}(y) \geqq \beta$ for each cyclic element $y \in Y$.

Proof. By way of contradiction, suppose that there exists $\beta \in \mathbb{C}$ ard $-\{0\}$ such that for every $Y \in Z_{n}^{1} \cap \mathcal{K}, \mathrm{w}(y)<\beta$ for at least one cyclic element $y \in Y$.

Let us consider an algebra $D \in \mathcal{Z}_{n}^{1}$ such that w $(d)=\beta$ for each cyclic element $d \in$ $D$. According to the assumption, $D \notin \mathcal{K} \cap Z_{n}^{1}$, i.e., $D \notin \mathcal{K}$. By (2) of Definition 5, $D$ possesses a largest homomorphism-homogeneous subalgebra belonging to $\mathcal{K}$; denote it by $B$. Then $B \neq \varnothing$ and $B \not \mathbb{Z}_{n}$ because $\mathcal{K} \cap \mathcal{Z}_{n}^{1} \neq \varnothing$. Also, $B \neq D$ because $D \notin \mathcal{K}$. 
Let $d_{i}, i=0,1, \ldots, n-1$, be the cyclic elements of $D$ such that $d_{i}=f\left(d_{i-1}\right)$ $(i-1$ is counted $\bmod n)$. For $d_{i}(i=0,1, \ldots, n-1)$ denote by $D_{i}$, the set of all acyclic elements $x \in D$ such that $f(x)=d_{i}$, i.e., $D_{i}=f^{-1}\left(d_{i}\right)-\left\{d_{i-1}\right\}$.

Now we show that if for some $i=0,1, \ldots, n-1$ there exists $x \in D_{i}$ such that $x \notin B$ then $D_{i} \cap B=\varnothing$.

If $\left|D_{i}\right|=1$ then the assumption is obvious.

Let $\left|D_{i}\right| \geqq 2$. Assume that $x \notin B, x \in D_{i}$ for some $i \in\{0,1, \ldots, n-1\}$ and suppose, to the contrary, that there exists an element $y$ such that $y \in B \cap D_{i}$. Then $C=(B-$ $\{y\}) \cup\{x\}$ is a subalgebra of $D$ and $C \cong B$. By (0) of Definition 5, $C \in \mathcal{K}$. Hence, $B$ cannot be the largest homomorphism-homogeneous subalgebra of $D$ belonging to $\mathcal{K}$, a contradiction.

Because $B \neq D$ and $B \not \mathbb{Z}_{n}$ there exists at least one $i \in\{0,1, \ldots, n-1\}$ such that $D_{i} \cap B=\varnothing$. On the other hand, for some $j \neq i(j \in\{0,1, \ldots, n-1\}), \varnothing \neq D_{j} \subseteq B$. Otherwise, the assumption that $D_{i} \cap B=\varnothing$ for each $i=0,1, \ldots, n-1$, would imply that $B \cong \mathbb{Z}_{n}$, which is a contradiction.

Next we show that $D$ possesses a subalgebra $C$ such that $C \cong B$ and $C \nsubseteq B$, $(C \neq B)$. Then this will be a contradiction with (2) of Definition 5, and will complete the proof.

From the definition of $D$ it follows that there exists an automorphism $\varphi_{0}$ of $D$ such that $\varphi_{0}(j)=i$. Since $B$ is a subalgebra of $D$, the mapping $\varphi=\varphi_{0} \uparrow B$ is an embedding of $B$ into $D$ such that $\varphi(j)=i$. Put $C=\varphi(B)$. Then $\varphi\left(D_{j}\right)=D_{i}$. Let $c \in D_{i}$. Then $c \in \varphi(B)=C$, but $c \notin \varnothing=B \cap D_{i}$. This implies that $C \nsubseteq B$. By condition (0) of Definition 5 we have $C \in \mathcal{K}$, which completes the proof.

Lemma 7. Let $\mathcal{K}$ be a class of monounary algebras such that $\mathcal{Z}_{n}^{1} \cap \mathcal{K} \neq \varnothing$ for some $n \in \mathbb{N}$. If $\mathcal{K}$ is an Hhr-class then $\mathfrak{Z}_{n}^{1} \subseteq \mathcal{K}$.

Proof. Assume that $\mathcal{K}$ is an Hhr-class and suppose, to the contrary, that $\mathcal{Z}_{n}^{1} \nsubseteq \mathcal{K}$. Then there exists an algebra $A \in \mathcal{Z}_{n}^{1}$ such that $A \notin \mathcal{K}$. Let $\beta=\max \{\mathrm{w}(a): a \in A, a$ is cyclic $\}$. Denote by $B$, an algebra such that $B \in \mathcal{Z}_{n}^{1}$ and $\mathrm{w}(b)=\beta$ for each cyclic element $b \in B$. Then according to Lemma $6, B \in \mathcal{K}$. Observe, that $B$ contains a homomorphism-homogeneous subalgebra $X$ such that $X \cong A$. Because $\mathcal{K}$ is an Hhr-class, we get that $A \in \mathcal{K}$, which is a contradiction.

Definition 7. For a class $\mathcal{K}$ of monounary algebras denote

$$
P(\mathcal{K})=\left\{i \in \mathbb{N}: Z_{i}^{1} \cap \mathcal{K} \neq \varnothing\right\}
$$

and $Q(\mathcal{K})=\left\{i \in \mathbb{N}: \mathcal{Z}_{i} \cap \mathcal{K} \neq \varnothing\right\}$.

Note that if $\mathcal{K}$ is an Hhr-class of monounary algebras then obviously, $P(\mathcal{K}) \subseteq$ $Q(\mathcal{K})$, and if $P(\mathcal{K}) \neq \varnothing$ then according to Lemma 7 we have:

$$
P(\mathcal{K})=\left\{i \in \mathbb{N}: Z_{i}^{1} \cap \mathcal{K} \neq \varnothing\right\}=\left\{i \in \mathbb{N}: Z_{i}^{1} \subseteq \mathcal{K}\right\} .
$$

Further, $Q(\mathcal{K})=\left\{i \in \mathbb{N}: Z_{i} \cap \mathcal{K} \neq \varnothing\right\}=\left\{i \in \mathbb{N}: Z_{i} \subseteq \mathcal{K}\right\}$. 
Lemma 8. Let $\mathcal{K}$ be an Hhr-class of monounary algebras such that $Q(\mathcal{K})=$ $Q \neq \varnothing$. Then $\sum_{i \in Q} \alpha_{i} \cdot \mathbb{Z}_{i} \in \mathcal{K}$ for each $\alpha_{i} \in \mathbb{C}$ ard.

Proof. By way of contradiction, suppose that there exists $\alpha_{i} \in \mathbb{C}$ ard, $i \in Q$, such that $D=\sum_{i \in Q} \alpha_{i} \cdot \mathbb{Z}_{i} \notin \mathcal{K}$. Observe that $D$ has to contain at least two connected components, since otherwise the assumption $D=\mathbb{Z}_{i}$ for some $i \in Q$ would imply that $\mathcal{Z}_{i} \nsubseteq \mathcal{K}$, a contradiction. By (2) of Definition $5, D$ possesses a largest homomorphism-homogeneous subalgebra belonging to $\mathcal{K}$, denote it by $D \mathcal{K}$. Since $D \notin \mathcal{K}$, we have $D \mathcal{K} \neq D$. Then $D$ possesses a subalgebra $C \cong \mathbb{Z}_{j}$ for some $j \in Q$ such that $C \nsubseteq D_{\mathcal{K}}$. From the assumption that $j \in Q=\left\{i \in \mathbb{N}: \mathcal{Z}_{i} \subseteq \mathcal{K}\right\}$ it follows that $C \in \mathcal{K}$ and note that $C$ is a homomorphism-homogeneous subalgebra of $D$. Then $D_{\mathcal{K}}$ is incomparable with $C$. Thus $D_{\mathcal{K}}$ cannot be the largest homomorphismhomogeneous subalgebra of $D$ and this is a contradiction.

Lemma 9. Let $\mathcal{K}$ be an Hhr-class with $Q(\mathcal{K}) \neq \varnothing$. Then $\mathcal{K}$ contains each $A$ such that if $B$ is a connected component of $A$ then either $B \in Z_{i}^{1}$ for some $i \in P(\mathcal{K})$ or $B \in \mathcal{Z}_{j}$ for some $j \in Q(\mathcal{K})$.

Proof. If $P(\mathcal{K})=\varnothing$ then the theorem follows from Lemma 8. Thus suppose that $P(\mathcal{K}) \neq \varnothing$ and let $A$ be a monounary algebra such that if $B$ is a connected component of $A$ then either $B \in Z_{i}^{1}$ for some $i \in P(\mathcal{K})$ or $B \in Z_{j}$ for some $j \in Q(\mathcal{K})$. If $A$ consists of cycles then the theorem follows from Lemma 8. So, suppose that there exists at least one acyclic element of $A$. By way of contradiction, suppose that $A \notin \mathcal{K}$. By (2) of Definition 5, $A$ possesses the largest homomorphism-homogeneous subalgebra $A \mathcal{K}$ such that $A \mathcal{K} \in \mathcal{K}$. Denote by $B$ the subalgebra of $A$ such that $B$ consists of all cyclic elements of $A$. According to Lemma 8 and (0) of Definition 5, $B \in \mathcal{K}$. Furthermore, $B$ is a homomorphism-homogeneous subalgebra of $A$ and then $B \subseteq A_{\mathcal{K}}$. Because $B \subseteq A_{\mathcal{K}} \neq A$, there exists an acyclic element $x \in A-A_{\mathcal{K}}$. Denote by $X$ that connected component of $A$ which contains the acyclic element $x$. Note that $X \in Z_{i}^{1}$ for some $i \in P(\mathcal{K})$ and $X$ is a homomorphism-homogeneous subalgebra of $A$. According to Lemma $7, Z_{i}^{1} \subseteq \mathcal{K}$, so, $X \in \mathcal{K}$. Since $X \nsubseteq A_{\mathcal{K}}, A_{\mathcal{K}}$ is incomparable with $X$. Thus $A \mathcal{K}$ cannot be the largest homomorphism-homogeneous subalgebra of $A$ such that $A_{\mathcal{K}} \in \mathcal{K}$, a contradiction.

Lemma 10. Let $\mathcal{K}$ be an Hhr-class such that $\mathbb{Z} \in \mathcal{K}$. Then $\mathcal{K}$ contains each $A$ such that if $B$ is a connected component of $A$ then either $B \in Z_{i}$ for some $i \in Q(\mathcal{K})$ or every branch in $B$ is infinite and $B$ possesses no cycle.

Proof. Let us consider a monounary algebra $A$ such that if $B$ is a connected component of $A$ then either $B \in \mathcal{Z}_{i}$ for some $i \in Q(\mathcal{K})$ or every branch in $B$ is infinite and $B$ possesses no cycle. Assume that $\mathcal{K}$ is an Hhr-class and suppose, to the contrary, that $A \notin \mathcal{K}$. By (2) of Definition 5, $A$ possesses the largest homomorphismhomogeneous subalgebra $A \mathcal{K}$ belonging to $\mathcal{K}$. Obviously, $\varnothing \neq A \mathcal{K} \neq A$. Consider 
the following two cases according to the definition of $Q(\mathcal{K})$.

Case 1: Let $Q(\mathcal{K})=\varnothing$. Because $A_{\mathcal{K}} \neq A, A$ possesses a subalgebra $X$ such that $X \cong \mathbb{Z}$ and $X \nsubseteq A_{\mathcal{K}}$. The subalgebra $X$ of $A$ is homomorphism-homogeneous and by (0) of Definition 5, $X \in \mathcal{K}$. Since $X \nsubseteq A_{\mathcal{K}}, A_{\mathcal{K}}$ is incomparable with $X$. Thus $A \mathcal{K}$ cannot be the largest homomorphism-homogeneous subalgebra of $A$ such that $A_{\mathcal{K}} \in \mathcal{K}$, a contradiction.

Case 2: Let $Q(\mathcal{K}) \neq \varnothing$. If $A$ possesses a subalgebra $X$ such that $X \cong \mathbb{Z}$ and $X \nsubseteq A_{\mathcal{K}}$, then as in the previous case we get a contradiction. So, suppose that $A_{\mathcal{K}}$ possesses all acyclic elements of $A$. Then $A-A \mathcal{K}$ contains an $i$-element cycle for some $i \in Q(\mathcal{K})$; denote it by $Y$. Because $Z_{i} \subseteq \mathcal{K}$ for $i \in Q(\mathcal{K}), Y \in \mathcal{K}$. Note that $Y$ is a homomorphism-homogeneous subalgebra of $A$. But then $A_{\mathcal{K}}$ is incomparable with $Y$, which is a contradiction and completes the proof.

The following two theorems present a characterization of Hhr-classes; in the first one we suppose that $\mathbb{Z}$ does not belong to the corresponding class $\mathcal{K}$ and that $Q(\mathcal{K}) \neq$ $\varnothing$, while in the second one, $\mathbb{Z} \in \mathcal{K}$.

Theorem 4. Let $\mathcal{K}$ be a class of monounary algebras such that $\mathbb{Z} \notin \mathcal{K}$ and $P(\mathcal{K}) \subseteq Q(\mathcal{K}) \neq \varnothing$. Next, let $\mathcal{K}$ satisfy (0) of Definition 5. Then $\mathcal{K}$ is an Hhrclass if and only if the following conditions hold:

(a) $\varnothing \in \mathcal{K}$.

(b) For every $A \in \mathcal{K}$, if $B$ is a connected component of $A$ then $B \in Z_{i}^{1}$ for some $i \in P(\mathcal{K})$ or $B \in Z_{j}$ for some $j \in Q(\mathcal{K})$ or $B$ possesses neither cycle nor subalgebra isomorphic to $\mathbb{Z}$.

(c) The class $\mathcal{K}$ includes all algebras $A$ such that if $B$ is a connected component of $A$ then either $B \in Z_{i}^{1}$ for some $i \in P(\mathcal{K})$ or $B \in Z_{j}$ for some $j \in Q(\mathcal{K})$.

Proof. " $\Rightarrow$ " Suppose that $\mathcal{K}$ is an Hhr-class. Then the condition (a) is obviously valid. From the assumption that $\mathbb{Z} \notin \mathcal{K}$ it follows that the class $\mathcal{K}$ does not contain an algebra having a subalgebra which is isomorphic to $\mathbb{Z}$.

By Corollary 3 , the class $\mathcal{K}$ does not contain a monounary algebra possessing a regular subalgebra of height greater than one. Obviously, if some $i \notin P(\mathcal{K})$ then the class $\mathcal{K}$ does not contain a monounary algebra possessing a subalgebra from $Z_{i}^{1}$. Also, if $j \notin Q(\mathcal{K})$ then $\mathcal{K}$ does not contain an algebra possessing a $j$-element cycle. Hence, the condition (b) is valid. The condition (3) results from Lemma 9.

" $\Leftarrow$ " Suppose that the conditions (a), (b) and (c) are true. Clearly, (0) of Definition 5 holds. Let $A \in \mathcal{K}$, and let $B$ be an arbitrary homomorphism-homogeneous subalgebra of it. If $B=\varnothing$ then by the condition (a), $B \in \mathcal{K}$. Now suppose that $B \neq \varnothing$. Then by Theorem 3, if $S$ is a connected component of $B$ then either $S \in Z_{i}$ for some $i \in Q(\mathcal{K})$ or $S \in Z_{j}^{1}$ for some $j \in P(\mathcal{K})$. Thus $B \in \mathcal{K}$ in view of the condition (c). Hence (1) of Definition 5 holds. Next, we show that $\mathcal{K}$ satisfies (2) of Definition 5. Let $D$ be an arbitrary monounary algebra. At first suppose that $D$ contains neither subalgebra belonging to $Z_{i}^{1}$ for each $i \in P(\mathcal{K})$ nor subalgebra belonging 
to $\mathcal{Z}_{j}$ for each $j \in Q(\mathcal{K})$. Then the largest subalgebra of $D$ belonging to $\mathcal{K}$ is $\varnothing$. Now suppose that $D$ possesses the largest non-empty subalgebra $E$ such that if $X$ is a connected component of $E$ then either $X \in \mathcal{Z}_{i}^{1}$ for some $i \in P(\mathcal{K})$ or $X \in \mathcal{Z}_{j}$ for some $j \in Q(\mathcal{K})$. By Theorem 3,E is a homomorphism-homogeneous algebra and by the condition (c), $E \in \mathcal{K}$. Then $E$ is the largest homomorphism-homogeneous subalgebra of $D$ belonging to $\mathcal{K}$. Thus (2) of Definition 5 holds for the class $\mathcal{K}$ and $\mathcal{K}$ is an Hhr-class.

Theorem 5. Let $\mathcal{K}$ be a class of monounary algebras such that $\mathbb{Z} \in \mathcal{K}$ and let $\mathcal{K}$ satisfy (0) of Definition 5. Then $\mathcal{K}$ is an Hhr-class if and only if

(a) $\varnothing \in \mathcal{K}$.

(b) For all $A \in \mathcal{K}$, if $B$ is a connected component of $A$ then $B$ possesses a subalgebra isomorphic to $\mathbb{Z}$ or $B \in Z_{i}$ for some $i \in Q(\mathcal{K})$ or $B$ possesses neither cycle nor subalgebra isomorphic to $\mathbb{Z}$.

(c) The class $\mathcal{K}$ includes all monounary algebras $A$ such that if $B$ is a connected component of $A$ then either $B \in \mathcal{Z}_{i}$ for some $i \in Q(\mathcal{K})$ or every branch in $B$ is infinite and $B$ possesses no cyclic element.

Proof. " $\Rightarrow$ "Suppose that $\mathcal{K}$ is an Hhr-class. Then the condition (a) is obviously valid.

From Lemma 5 it follows, that $\mathcal{K}$ does not contain an algebra possessing a regular subalgebra of nonzero height. Thus $P(\mathcal{K})=\varnothing$. Also, $\mathcal{K}$ does not contain an algebra with an $i$-element cycle for $i \notin Q(\mathcal{K})$. Hence the condition (b) holds.

According to Lemma 10, the condition (c) is valid.

$" \Leftarrow$ " Assume that the conditions (a), (b) and (c) are true. Obviously, (0) of Definition 5 holds. Let $A \in \mathcal{K}$ and $B$ be its any homomorphism-homogeneous subalgebra. If $B=\varnothing$ then by the condition (a), $B \in \mathcal{K}$. So, suppose that $B \neq \varnothing$. Then according to Theorem 3, if $S$ is a connected component of $B$ then either $S \in \mathcal{Z}_{i}$ for some $i \in Q(\mathcal{K})$ or every branch in $S$ is infinite and $S$ possesses no cycle. Hence, $B \in \mathcal{K}$ in view of the condition (c). Thus (1) of Definition 5 holds.

Next, we show that $\mathcal{K}$ satisfies (2) of Definition 5. Let $D$ be an arbitrary monounary algebra. Assume that $D$ possesses neither $i$-element cycle for $i \in Q(\mathcal{K})$ nor subalgebra isomorphic to $\mathbb{Z}$. Then $\varnothing$ is the largest homomorphism-homogeneous subalgebra of $D$ such that $\varnothing \in \mathcal{K}$. Now suppose that $D$ possesses the largest nonempty subalgebra $E$ such that if $X$ is a connected component of $E$ then either every branch in $X$ is infinite and $X$ possesses no cycle or $X \in Z_{i}$ for some $i \in Q(\mathcal{K})$. By Theorem 3, $E$ is a homomorphism-homogeneous algebra and by the condition (c), $E \in \mathcal{K}$. Then $E$ is the largest homomorphism-homogeneous subalgebra of $D$ belonging to $\mathcal{K}$. Thus (2) of Definition 5 holds and $\mathcal{K}$ is an Hhr-class.

Let $\mathcal{K}$ be an Hhr-class. Denote by $\mathscr{H}^{*}(\mathcal{K})$ the subclass of $\mathcal{K}$ such that $\mathscr{H}^{*}(\mathcal{K})$ consists of all homomorphism-homogeneous algebras of $\mathcal{K}$. Then $\mathscr{H}^{*}(\mathcal{K})$ is called a homomorphism-homogeneous part of $\mathcal{K}$. Note that $\mathscr{H}^{*}(\mathcal{K})$ is an Hhr-class. 
Corollary 5. Let $\mathcal{K}$ be an Hhr-class such that $\mathbb{Z} \notin \mathcal{K}$ and $Q(\mathcal{K}) \neq \varnothing$. Then $\mathscr{H}^{*}(\mathcal{K})$ is the class of all monounary algebras $A$ such that if $B$ is a connected component of $A$ then either $B \in Z_{i}^{1}$ for some $i \in P(\mathcal{K})$ or $B \in Z_{j}$ for some $j \in Q(\mathcal{K})$.

Proof. The assertion follows from Theorems 3 and 4.

Corollary 6. Let $\mathcal{K}$ be an Hhr-class such that $\mathbb{Z} \in \mathcal{K}$. Then $\mathcal{H}^{*}(\mathcal{K})$ is the class of all monounary algebras $A$ such that if $B$ is a connected component of $A$ then either $B \in Z_{i}$ for some $i \in Q(\mathcal{K})$ or every branch in $B$ is infinite and $B$ possesses no cyclic element.

Proof. The assertion follows from Theorems 3 and 5.

We will finish with a comparison of hr-classes and Hhr-classes as follows. All homogeneous algebras are homomorphism-homogeneous, too. But, in general an hr-class fails to be an Hhr-class. Assume that $\mathcal{K}$ is a class of monounary algebras which is closed with respect to isomorphisms, contains $\varnothing$ and that for all $A \in \mathcal{K}, A$ possesses neither cycles nor subalgebra isomorphic to $\mathbb{Z}$. Then obviously, $\mathcal{K}$ is an hr-class and Hhr-class, too. The following assertions are consequence of Theorems 2 and 4.

Proposition 1. Let $\mathcal{K}$ be an hr-class such that $\mathbb{Z}_{n} \in \mathcal{K}$ for some $n \in \mathbb{N}$. Then an hr-class $\mathcal{K}$ is an Hhr-class if and only if $\mathcal{K}$ satisfies the following conditions:

(1) $\mathcal{K}$ does not contain a monounary algebra possessing a regular subalgebra of height greater than one

(2) if $\mathcal{K}$ contains an algebra $A$ having a subalgebra $B \in \mathcal{Z}_{n}^{1}$ then $\mathcal{K}$ contains all algebras $C$ such that whenever $X$ is a connected component of $C$ then $X \in \mathcal{Z}_{n} \cup \mathcal{Z}_{n}^{1}$.

Proposition 2. Let $\mathcal{K}$ be an Hhr-class. Then $\mathcal{K}$ is an hr-class if and only if $\mathcal{K}$ does not contain a monounary algebra possessing a subalgebra isomorphic to $\mathbb{Z}$ and $|Q(\mathcal{K})|<2$.

\section{TORSION CLASSES OF MONOUNARY ALGEBRAS CONCERNING HOMOGENEITY}

In this section we will briefly characterize classes of monounary algebras which are torsion classes with respect to the homogeneity or homomorphism-homogeneity of monounary algebras.

Definition 8. A non-empty class $\mathcal{K}$ of monounary algebras will be called a homogeneous torsion class (for short, ht-class) if it satisfies the following conditions:

(0) $\mathcal{K}$ is closed with respect to homomorphisms,

(1) whenever $A \in \mathcal{K}$ and $B$ is an homogeneous subalgebra of $A$, then $B \in \mathcal{K}$,

(2) for each monounary algebra $D$ there exists its largest homogeneous subalgebra belonging to $\mathcal{K}$. 
For a class $\mathcal{K}$ of monounary algebras we will use the notions $P(\mathcal{K})$ and $Q(\mathcal{K})$ from the previous section. Note that from Theorem 2 it follows that if $\mathcal{K}$ is an hr-class then $|Q(\mathcal{K})|<2$.

Theorem 6. Let $\mathcal{K}$ be a class of monounary algebras closed with respect to homomorphisms. Then $\mathcal{K}$ is an ht-class if and only if $\mathcal{K}$ is an hr-class such that either $Q(\mathcal{K})=\varnothing$ or $Q(\mathcal{K})=\{1\}$.

Proof. Suppose that $\mathcal{K}$ is an ht-class. Then obviously, $\mathcal{K}$ is an hr-class. The theorem trivially holds in the case when $|Q(\mathcal{K})|=0$ or $Q(\mathcal{K})=\{1\}$. Next, suppose that $|Q(\mathcal{K})|=1$ and $1 \notin Q(\mathcal{K})$. The assumption that $n \in Q(\mathcal{K})$ for some $n \in$ $\mathbb{N}-\{1\}$, would imply that $\mathbb{Z}_{1} \in \mathcal{K}$ because $\mathbb{Z}_{1}$ is a homomorphic image of $\mathbb{Z}_{n}$. But then $\{1, n\} \subseteq Q(\mathcal{K})$, which is a contradiction. Conversely, it is clear that $\mathcal{K}$ satisfies each condition of Definition 8, i.e., $\mathcal{K}$ is an ht-class.

Definition 9. A non-empty class $\mathcal{K}$ of monounary algebras will be called a homomorphism-homogeneous torsion class (for short, Hht-class) if it satisfies the following conditions:

(0) $\mathcal{K}$ is closed with respect to homomorphisms,

(1) whenever $A \in \mathcal{K}$ and $B$ is a homomorphism-homogeneous subalgebra of $A$, then $B \in \mathcal{K}$,

(2) for each monounary algebra $D$ there exists its largest homomorphism-homogeneous subalgebra belonging to $\mathcal{K}$.

It is easy to see the following assertion. Let $\mathcal{K}$ be a class of monounary algebras such that $\mathcal{K}$ is closed with respect to homomorphisms and $\varnothing \in \mathcal{K}$. Next, assume that each $A \in \mathcal{K}$ possess neither cycle nor subalgebra isomorphic to $\mathbb{Z}$. Then, by definition, $\mathcal{K}$ is an Hht-class.

Lemma 11. Let $\mathcal{K}$ be an Hht-class. Then $\mathcal{K}$ fails to contain a monounary algebra possessing a subalgebra which is isomorphic to $\mathbb{Z}$.

Proof. By way of contradiction suppose that $\mathcal{K}$ contains an algebra with a subalgebra isomorphic to $\mathbb{Z}$. Since $\mathcal{K}$ is an Hht-class, $\mathbb{Z} \in \mathcal{K}$. Observe that a regular monounary algebra which possesses only one infinite branch is a homomorphic image of $\mathbb{Z}$. By $(0)$ of Definition 9, this regular algebra belongs to $\mathcal{K}$. According to Lemma 5, $\mathcal{K}$ fails to be an Hhr-class which is a contradiction and completes the proof.

Notation 2. Let for $i \in \mathbb{N}$ be $D_{i}=\{j \in \mathbb{N}: j \mid i\}$. Then for a class $\mathcal{K}$ of monounary algebras denote $Q^{*}(\mathcal{K})=\bigcup_{i \in Q(\mathcal{K})} D_{i}$ and $P^{*}(\mathcal{K})=\bigcup_{i \in P(\mathcal{K})} D_{i}$.

According to Lemma $11, \mathbb{Z}$ does not belong to an Hht-class. Therefore the proof of the following characterization theorem is analogous to the proof of Theorem 4. It suffices to replace $Q(\mathcal{K})$ by $Q^{*}(\mathcal{K})$ and $P(\mathcal{K})$ by $P^{*}(\mathcal{K})$. 
Theorem 7. Let $\mathcal{K}$ be a class of monounary algebras such that $P(\mathcal{K}) \subseteq Q(\mathcal{K}) \neq$ $\varnothing$. Next, let $\mathcal{K}$ satisfy (0) of Definition 9. Then $\mathcal{K}$ is an Hht-class if and only if the following conditions hold:

(a) $\varnothing \in \mathcal{K}$.

(b) For every $A \in \mathcal{K}$, if $B$ is a connected component of $A$ then $B \in Z_{i}^{1}$ for some $i \in P^{*}(\mathcal{K})$ or $B \in Z_{j}$ for some $j \in Q^{*}(\mathcal{K})$ or $B$ possesses neither cycle nor subalgebra isomorphic to $\mathbb{Z}$.

(c) The class $\mathcal{K}$ includes all algebras $A$ such that if $B$ is a connected component of $A$ then either $B \in Z_{i}^{1}$ for some $i \in P^{*}(\mathcal{K})$ or $B \in Z_{j}$ for some $j \in Q^{*}(\mathcal{K})$.

\section{REFERENCES}

[1] W. Bartol, Dynamic programs of computations. Warszawa: PAN, 1974.

[2] S. Burris and H. P. Sankappanavar, A course in universal algebra, ser. Graduate Texts in Mathematics. New York - Heidelberg Berlin: Springer-Verlag, 1981, vol. 78.

[3] P. J. Cameron and J. Nešetřil, "Homomorphism-homogeneous relational structures," Comb. Probab. Comput., vol. 15, no. 1-2, pp. 91-103, 2006.

[4] J. Chvalina, Functional graphs, quasiordered sets and commutative hypergroups. Brno: Masaryk University, 1995.

[5] P. Conrad, "K-radical classes of lattice ordered groups," Algebra, Proc. Conf., Carbondale 1980, Lect. Notes Math., vol. 848, pp. 186-207, 1981.

[6] I. Dolinka and D. Mašulović, "Remarks on homomorphism-homogeneous lattices and semilattices," Monatsh. Math., vol. 164, no. 1, pp. 23-37, 2011.

[7] D. Jakubíková-Studenovská and J. Pócs, Monounary algebras. Košice: Pavol Jozef Šafárik University, 2009.

[8] J. Jakubik, "Radical mappings and radical classes of lattice ordered groups," Symp. math. 21, Gruppi e Anelli ordin., Convegno 1975, pp. 451-477, 1977.

[9] J. Jakubík, "Radical classes of generalized Boolean algebras," Czech. Math. J., vol. 48, no. 2, pp. 253-268, 1998.

[10] J. Jakubík, "Radical classes of MV-algebras," Czech. Math. J., vol. 49, no. 1, pp. 191-211, 1999.

[11] D. Jakubiková-Studenovská, "Homogeneous monounary algebras," Czech. Math. J., vol. 52, no. 2, pp. 309-317, 2002.

[12] B. Jónsson, Topics in universal algebra, ser. Lecture Notes in Mathematics. Berlin-HeidelbergNew York: Springer-Verlag, 1972, vol. 250.

[13] E. Jungábel and D. M. sulović, "Homomorphism-homogeneous monounary algebras," Mathematica Slovaca, to appear.

[14] P. Körtesi, S. Radeleczki, and S. Szilágyi, "Congruences and isotone maps on partially ordered sets," Math. Pannonica, vol. 16, no. 1, pp. 39-55, 2005.

[15] W. Lex and R. Wiegandt, "Torsion theory for acts," Stud. Sci. Math. Hung., vol. 16, pp. 263-280, 1981.

[16] J. Martinez, "Varieties of lattice-ordered groups," Math. Z., vol. 137, pp. 265-284, 1974.

[17] J. Martinez, "Torsion theory for lattice-ordered groups," Czech. Math. J., vol. 25, pp. 284-299, 1975.

[18] D. Mašulović, R. Nenadov, and N. Škorić, "On finite reflexive homomorphism-homogeneous binary relational systems," Discrete Math., vol. 311, no. 21, pp. 2543-2555, 2011. 
[19] D. Mašulović and M. Pech, "Oligomorphic transformation monoids and homomorphismhomogeneous structures," Fundam. Math., vol. 212, no. 1, pp. 17-34, 2011.

[20] H. Neumann, Varieties of groups. (Ergebnisse der Mathematik und ihrer Grenzgebiete, Bd. 37.). Berlin-Heidelberg-New York: Springer-Verlag, 1967.

[21] M. Novotný, "Mono-unary algebras in the work of Czechoslovak mathematicians," Arch. Math., Brno, vol. 26, no. 2-3, pp. 155-164, 1990.

[22] R. S. Pierce, "Some questions about complete Boolean algebras," Proc. Sympos. Pure Math., vol. 2, pp. 129-140, 1961.

[23] M. Rusinov and P. Schweitzer, "Homomorphism-homogeneous graphs," J. Graph Theory, vol. 65, no. 3, pp. 253-262, 2010.

[24] J. Szigeti, "On well-orderd mono-unary algebras," Order, vol. 7, no. 1, pp. 77-81, 1990.

[25] D.-R. Ton, "Radical classes of $\ell$-groups," Internat. J. Math. and Math. Sci., vol. 17, no. 1, pp. 129-142, 1994

[26] J. von Neumann, "Lectures on continuous geometries," Princeton University Press, Princeton, pp. 1936-1937, 1967.

Authors' addresses

\section{Zuzana Farkasová}

P.J.Šafárik University, Institute of Mathematics, Jesenná 5, 04154 Košice, Slovakia

E-mail address: zuzana.farkasova@student.upjs.sk

\section{Danica Jakubíková-Studenovská}

P.J.Šafárik University, Institute of Mathematics, Jesenná 5, 04154 Košice, Slovakia

E-mail address: danica.studenovska@upjs.sk 